\title{
Silicon Photomultiplier Readout of a Scintillating Noble Gas Detector for Homeland Security
}

\author{
Massimo Caccia, Valery Chmill, Alexander Martemiyanov, Romualdo Santoro, Rico Chandra, Giovanna Davatz, \\ Ulisse Gendotti
}

\begin{abstract}
Detectors based on scintillation by high pressure ${ }^{4} \mathrm{He}$ are a viable technology for instruments against the illicit trafficking of nuclear material. A design based on the use of solid state photodetectors is presented in this paper and the preliminary qualification discussed.
\end{abstract}

Keywords-Silicon Photo-Multipliers, Scintillating Noble Gases, Neutron detection, Neutron-Gamma discrimination

\section{INTRODUCTION}

$\mathbf{I}$ LLICIT trafficking of radioactive material represents a major threat and a priority in the international security program [1], emphasizing the relevance of Special Nuclear Material (SNM). SNM is defined by the U.S. Nuclear Regulatory Commission as the primary ingredient of improvised nuclear devices (IND) and it consists of [2]:

- Weapon Grade Plutonium (WGP), typically a mixture of ${ }^{239} \mathrm{Pu}(93 \%)$ and ${ }^{240} \mathrm{Pu}(6 \%)$;

- Weapon Grade Uranium (WGU), consisting of ${ }^{233} \mathrm{U}$ or Highly Enriched Uranium (HEU), i.e. an U sample enriched in the ${ }^{233} \mathrm{U}$ and ${ }^{235} \mathrm{U}$ isotopes;

characterized by the neutron and $\gamma$ radiation flux reported in Table I [3].

TABLE I: THE SPONTANEOUS GAMMA RAY AND NEUTRON EMISSIONS OF URANIUM AND PLUTONIUM. EMISSIONS ARE PER $K g \cdot s$, ENERGIES ARE IN $M e V$. FOR COMPARISON SIMILAR INFORMATION IS SHOWN FOR $1 \mu g r$ OF ${ }^{252} \mathrm{Cf}$.

\begin{tabular}{|c|c|c|c|c|c|}
\hline \multirow{2}{*}{ SNM } & \multirow{2}{*}{ Form } & \multicolumn{2}{|c|}{ Gamma-rays } & \multicolumn{2}{c|}{ Neutrons } \\
\cline { 3 - 6 } & & Energy & Intensity & Energy & Intensity \\
\hline \multirow{2}{*}{ Uranium } & \multirow{2}{*}{ Highly enriched } & 1.001 & $\leq 10^{4}$ & $\approx 2$ & 1 \\
& & 2.6 & $2.7 \times 10^{4}$ & & \\
\hline \multirow{2}{*}{ Plutonium } & Mixed Oxide & 0.769 & $10^{5}$ & $\approx 2$ & $\approx 5 \times 10^{5}$ \\
& Weapons grade & 0.769 & $2.3 \times 10^{5}$ & $\approx 2$ & $\approx 6 \times 10^{4}$ \\
\hline Cf-252 & & & & & $\approx 2 \times 10^{6}$ \\
\hline
\end{tabular}

The detection of SNM is not a trivial issues [4], irrespective from the fact IAEA [5] defines a significant quantity as $25 \mathrm{~kg}$ mass of WGP and $8 \mathrm{~kg}$ of WGU. The $\gamma$ ray flux can be significantly reduced by shielding and masked by emitting substances of common use in industry and by Natural Occurring Radioactive Material (NORM). At stand-off distance, the expected count rate from neutrons can drop at the natural background level, especially if neutrons are detected after

M. Caccia, V. Chmill, A. Martemiyanov, and R. Santoro are with the Dipartimento di Scienza e Alta Tecnologia, Universita' degli Studi dell'Insubria, 22100, Como, Italy (e-mail: massimo.caccia@uninsubria.it, romualdo.santoro@uninsubria.it).

R.Chandra,G. Davatz, U. Gendotti are with ARKTIS Radiation Detectors Ltd., Raffelstrasse 11, 8045, Zurich, Switzerland. thermalization. Nevertheless, neutron detection is a key issue in apparatus for security and it is usually performed by ${ }^{3} \mathrm{He}$ based systems because of the favorable capture cross section after thermalization. However, following the current shortage and the consequent cost increase [6], alternatives are being searched for [7]. ARKTIS, a spin-off company of ETH, the Zürich Polytechnical School, is exploiting a technique based on the detection of scintillating light in a high-pressure ${ }^{4} \mathrm{He}$ vessel [8]-[10]. This paper reports the first results on the use of Silicon Photomultipliers as photosensors in the ARKTIS baseline detector, expected to provide a cost effective solution with enhanced sensitivity.

\section{THE MODES-SNM PROJECT AND THE ARKTIS TECHNOLOGY}

MODES-SNM (MOdular DEtection System for Special Nuclear Material) is a project $^{1}$ approved by the European Commission within the VII Framework Program ${ }^{2}$ targeting the improvement of detection systems which could also be used by nuclear emergency responders. Enhancement is expected in terms of detection efficiency as well as portability and mobility, with a system capable to detect and identify the sources of interest. The consortium is developing a detector suite based on the ARKTIS technology, with solutions for thermal neutron detection based on a ${ }^{4} \mathrm{He}$ filled high pressure tube $\mathrm{Li}$ coated and a detector with spectrometric capability replacing ${ }^{4} \mathrm{He}$ with Xenon [11].

The ARKTIS technology relies on three features of He:

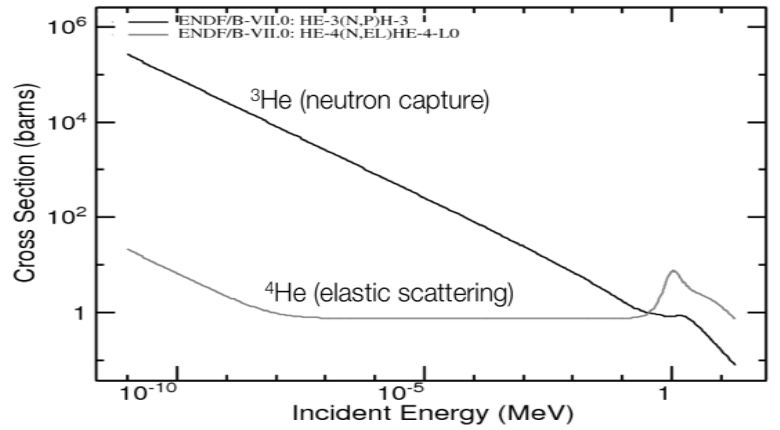

Fig. 1: The elastic scattering cross-section of ${ }^{4} \mathrm{He}$ exhibits a peak at around $1 \mathrm{MeV}$, matching the emission spectrum of fission neutrons rather well [12] .

\footnotetext{
${ }^{1}$ project id. no. 284842, http://www.modes-snm.eu/

${ }^{2}$ SECurity call 2011.1.5-1
} 
- as far as the interaction with neutrons, an elastic cross section comparable or exceeding the capture cross section for ${ }^{3} \mathrm{He}$ at energies above $1 \mathrm{MeV}$ (Fig. 1 [12]);

- light yield of 15000 photons / MeV of deposited energy and light emission at $70 \mathrm{~nm}$;

- scintillation characterized by two emitted components with decay time at the $n s$ and $\mu s$ level [13].

As shown in [10], fast neutron detection enhances significantly the system performance. In fact more than $95 \%$ of neutrons emitted by SNM are fast, i.e. with energy in excess of $200 \mathrm{KeV}$. Since the natural neutron background has a $1 / \mathrm{E}$ energy dependence [14], the lower neutron cross section in ${ }^{4} \mathrm{He}$ is over-compensated by the reduction in the natural background. This is resulting into a Minimum Detectable Level (MDL) of radiation at the $0.0049 \mu S v / h$ level for a False Alarm Rate not exceeding $3 \times 10^{-7}$, assuming a sensitivity of $150 \mathrm{cps} /(\mathrm{mSv} / \mathrm{h})$ on the base of laboratory results. This MDL value results to be lower with respect to a standard system based on thermal neutron detection and sets the ground for the proposed novel solution.

The baseline ARKTIS detector is shown in Fig. 2: ${ }^{4} \mathrm{He}$ is compressed at a pressure of $\approx$ 180bar in a cylinder having $44 \mathrm{~mm}$ diameter and $470 \mathrm{~mm}$ length. Scintillating UV light resulting from neutron scattering is converted into blue $(420 \mathrm{~nm})$ by a wave-length shifting material coating the cylinder. Blue light, diffused and reflected on the walls, is collected by Photo Multiplier Tubes (PMT) interfaced to the sensitive volume through a quartz window. The gas pressure represents a trade-off between sensitivity, safety regulations about high pressure vessels and a cost-effective design. The diameter matches the characteristics of the PMT in use and the length is again a compromise between uniformity of the response and overall sensitive volume.

High pressure vessel with embedded WLS coated reflector

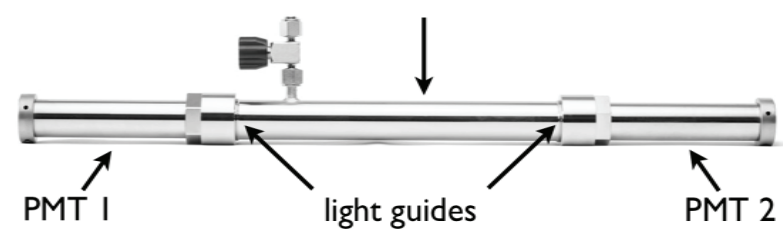

Fig. 2: Picture of the baseline ARKTIS detector

The overall system performance results by balancing detection neutron efficiency and background rejection, where the discrimination against $\gamma$ interaction is crucial to maintain the false alarm rate at the level required by the international security standards. In such a respect, the proposed detector benefits from several physical effects:

- the interaction probability of $1 \mathrm{MeV}$ photons is at the $1 \%$ level because of the low $\mathrm{Z}$ and the density;

- gamma induced signals are dominated by Compton scattering in the detector wall resulting in electrons ejected in the sensitive volume;
- the total energy loss by a recoiling Compton electron is expected not to exceed $750 \mathrm{KeV}$, to be compared to the maximum energy transfer from an incoming neutron to a ${ }^{4} \mathrm{He}$ nucleus with a kinematic limit at $64 \%$ of the neutrons energy prior to the interaction;

- the recoiling electron and the ${ }^{4} \mathrm{He}$ nucleus elastically scattered against a neutron are characterized by a different ionization density. This results in a different ratio between the short and long component of the emitted scintillation light, as shown in the exemplary event reported in Fig. 3 [10].
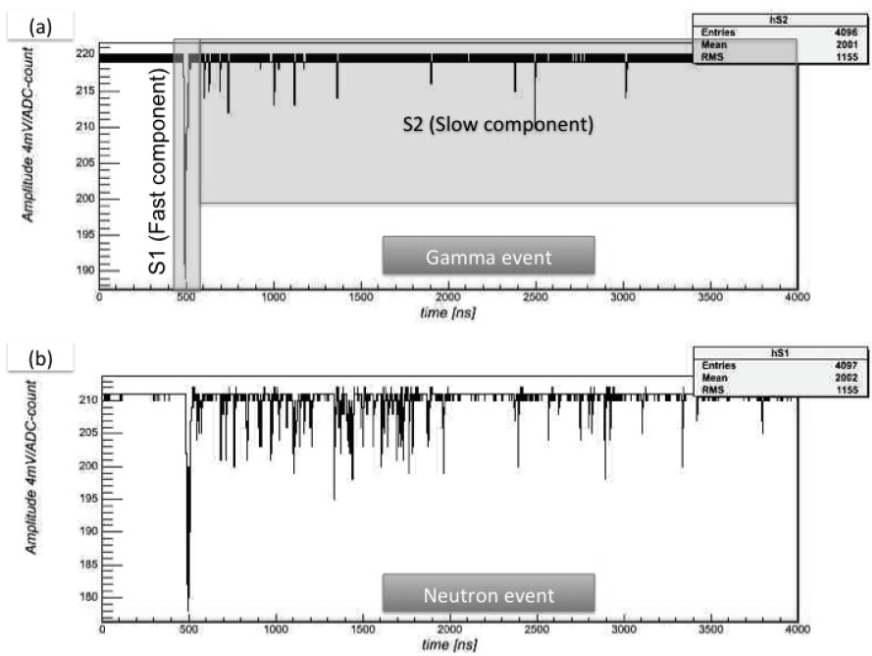

Fig. 3: (a) ${ }^{4} \mathrm{He}$ scintillation signals consist of a fast component with a decay time of few nanoseconds and a slow component of the order of a microsecond. Depending on the type of interaction the relative strengths of the two scintillation components are observed to be different. (a) shows a gamma event while (b) shows a neutron event.

Based on these effects, $\gamma$ rejection algorithms supported by an event-by-event pulse shape discrimination were implemented, resulting in a fission neutron detection efficiency at the $5 \%$ level over a $125 \mathrm{~cm}^{2}$ area while reducing to $0.03 \mathrm{cps}$ the rate due to $1 \mathrm{mSv} / \mathrm{hr}$ irradiation by ${ }^{60} \mathrm{Co}[10]$.

MODES-SNM is also addressing the optimization of the detector design in terms of mechanics, services and the photosensors. In such a respect, the use of Silicon Photomultipliers (SiPM) was considered since a significant improvement can be expected in terms of light collection and uniformity, robustness, compactness and simplification of the assembly.

The performance of the detector under study are primarily defined by the scintillation process and the light collection, determining the distribution in the number of detected photons for a particle interacting in the gas volume. The SiPM area, typically smaller with respect to PMT, may be expected to hamper their use. However, it should be considered that an average number of $\approx 60$ reflections are required for light to get on the PMT located at the cylinder ends in the standard configuration. Since the wavelength shifting diffusive material has a typical reflectivity at the $95 \%$ level, only $5 \%$ of the scintillation light will be collected by the PMT, with further losses at the 


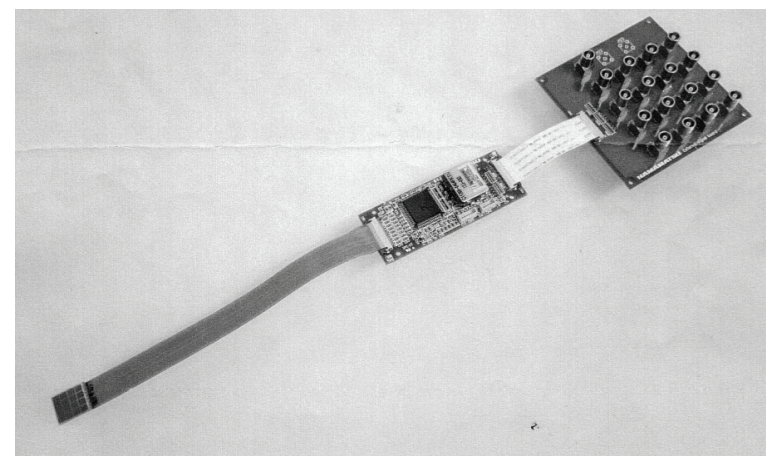

Fig. 4: Picture of the Hamamatsu MPPC connected to the readout board and to the signal output board.

optical interface. SiPM mounted inside the sensitive volume and properly located along the cylinder can lead to an improved light collection with the smaller area over-compensated by the reduced number of reflections required to convey the light on the sensitive surface. A Geant4 simulation supported this hypothesis and paved the way to the implementation reported in the following.

\section{CHARACTERIZATION OF THE SIPM ARRAY}

The results discussed in this paper are based on the SiPM array C11206-0404FB produced by Hamamatsu Photonics ${ }^{3}$, integrating $16(4 \times 4)$ separate elements on a unique monolithic substrate for an active area of $12 x 12 \mathrm{~mm}^{2}$ and a total number of 57600 cells (Fig. 4). The readout board shown in Fig. 4 provides the bias to the sensor with adjustable levels for every array element and implements a temperature feedback to stabilize the gain. The USB controlled board integrates a preamplifier and fans-out the individual channels and their sum. The first tests were aimed at measuring the minimum detectable light level. The SiPM array was illuminated by a calibrated pulsed LED source and the signal integrated in a pre-defined time window synchronized with the light emission. Scanning the light intensity, the separation with respect to the baseline fluctuations was used to measure the quantity of interest. The results in Fig. 5 shows a good peak separation at $\approx 60$ photons operating at $21.6^{\circ} \mathrm{C}$. This values qualifies the SiPM array in terms of sensitivity, since the Geant 4 simulation shows that the expected number of photons for the minimum deposited energy $(100 \mathrm{KeV})$ is expected to range between 240 and 480 depending on the wave-length shifting reflectivity.

The second tests were meant to assess the SiPM array performance in counting mode. The array was illuminated at tunable light intensity. The ratio between the LED pulsing frequency and the self-triggering count rate defined by a leading edge discriminator at tunable threshold was retained as a measurement of the detection efficiency for a given light level. This figure, together with the dark count rate, provides the input to the results reported in Fig. 6, showing the minimum interaction rate required to have a detected event

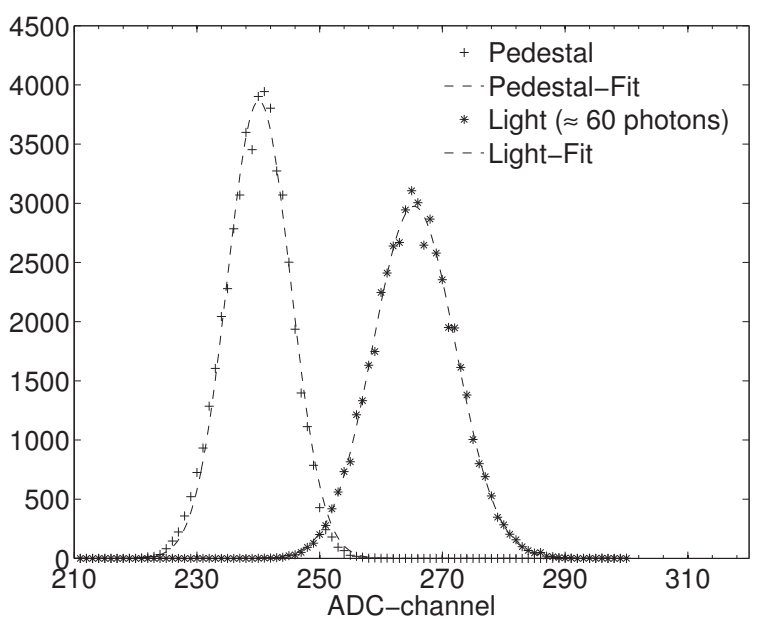

Fig. 5: Plot of the minimum detectable light $(\approx 60$ photons $)$ and baseline separation done with the SiPM array at $21^{\circ} \mathrm{C}$.

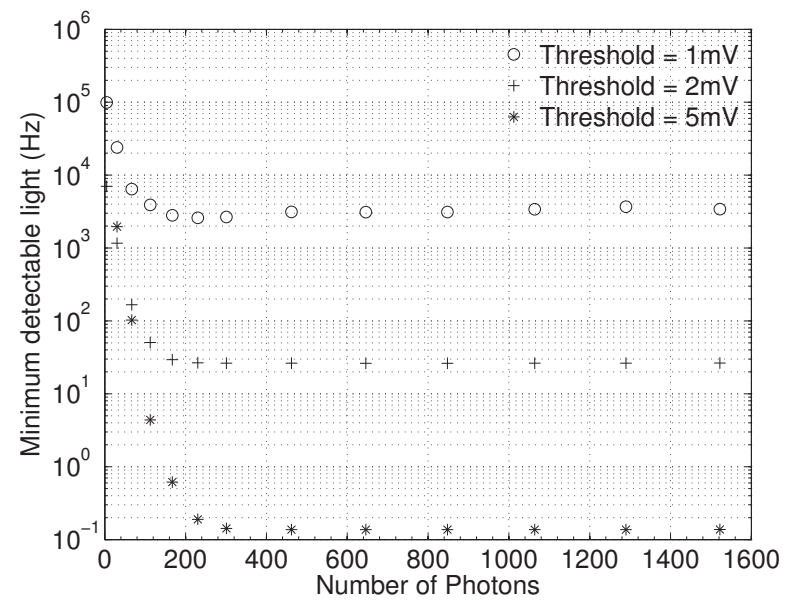

Fig. 6: Trends of the minimum interaction rate requested to have a $5 \sigma$ signal over noise separation. The three curves are obtained scanning the light intensity with three leading edge discriminating threshold.

rate exceeding by $5 \sigma$ the noise level. Referring once more to the simulation results, at 240 photons the threshold can be increased to $5 \mathrm{mV}$, so that an event rate at the $0.1 \mathrm{~Hz}$ level will allow a discrimination with a False Alarm Rate at the $6 \times 10^{-7}$, corresponding to $5 \sigma$.

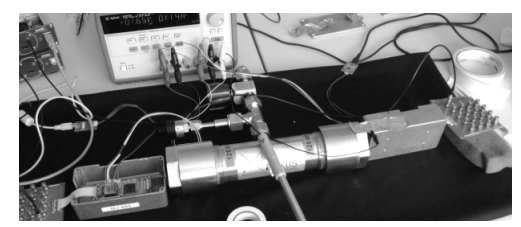

Fig. 7: Picture of the detector prototype with embedded SiPM arrays used for the measurements described in the paper. 


\section{MPPC INTEGRATION IN THE ARKTIS DETECTOR AND FIRST RESULTS}

An experimental proof of concept was performed integrating two MMPC arrays into a detector of reduced length $(19 \mathrm{~cm})$, equally spaced along the axis in order to maximize the uniformity in the collected light. Power voltage and signal routing was provided through flat kapton cables across feedthroughs at the detector ends (Fig. 7). The signals were amplified by the CAEN-SP5600 SiPM unit and processed by the CAENDT5720A digitizer. Tests were performed with ${ }^{4} \mathrm{He}$ at 140bar and analyzing the response of the system to $\gamma$ and neutron emitting sources $\left({ }^{60} \mathrm{Co}, 40.4 \mathrm{KBq}\right.$ activity; ${ }^{137} \mathrm{Cs}, 3.7 \mathrm{MBq}$ and ${ }^{252} \mathrm{Cf}, 37 \mathrm{KBq}$ ). The signals from interacting neutrons and $\gamma$ 's confirmed, in a qualitative way, the expected difference in terms of peak value, area and time development of the pulse (see for instance Fig. 9). A quantitative analysis was performed in two steps:

- evaluating different triggering schemes;

- studying off-line algorithms implemented on data recorded in minimum bias conditions.

\section{A. Real-time Neutron-c Discrimination}

The system performance was initially evaluated implementing a simple triggering scheme based on the signal amplitude, requiring the coincidence of the logical signals resulting from a leading edge discrimination of the pulses by both SiPM arrays. The recorded counting rates for the ambient background, ${ }^{60} \mathrm{Co}$ and ${ }^{252} \mathrm{Cf}$ in contact with the detector wall are reported in Fig. 8. The results in Table II shows that:

- the counting rate from cosmic rays and natural radioactivity can be reduced to the $50 \mathrm{mHz}$ level;

- in these conditions, the rate from ${ }^{60} \mathrm{Co}$ is lower by one order of magnitude with respect to events resulting from ${ }^{252} \mathrm{Cf}$, largely dominated by neutron interactions;

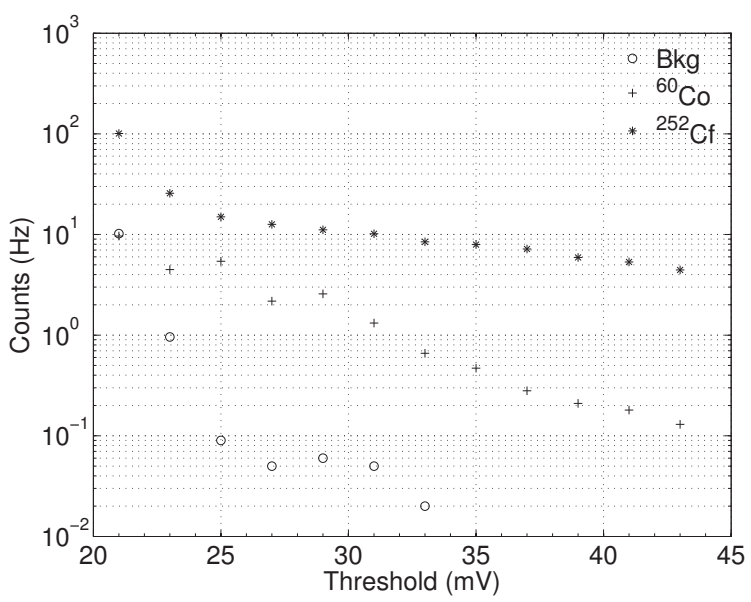

Fig. 8: Counting rates at different leading edge discriminating thresholds recorded without radioactive sources (ambient background), with ${ }^{60} \mathrm{Co}$ or with ${ }^{252} \mathrm{Cf}$ in contact with the detector wall.
TABLE II: COUNTING RATES IN DIFFERENT CONDITIONS IMPLEMENTING THE LEADING EDGE DISCRIMINATION COINCIDENCE. SOURCES WERE POSITIONED IN CONTACT WITH THE DETECTOR WALL.

\begin{tabular}{|c|c|c|c|}
\hline & \multicolumn{3}{|c|}{ Counting frequency $\nu[\mathrm{Hz}]$} \\
\cline { 2 - 4 } & No source & ${ }^{60} \mathrm{Co}$ & ${ }^{252} \mathrm{Cf}$ \\
{$[40.4 K B q]$} & {$[37 K B q]$} \\
\hline Coincidence & 0.05 & 1.32 & 10.18 \\
$\nu_{\text {coincidence }} / \nu_{\text {singlearm }}$ & $\approx 0.1$ & 0.35 & 0.39 \\
\hline
\end{tabular}
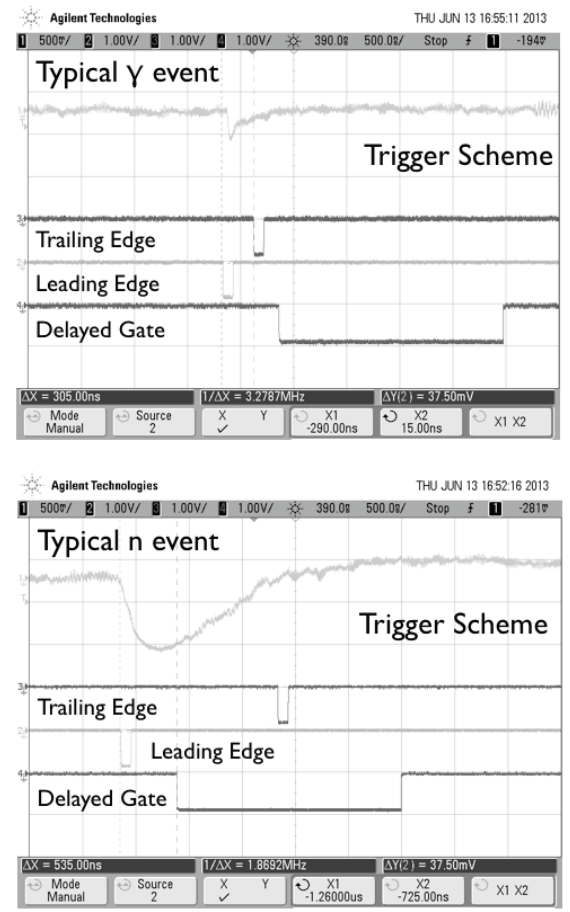

Fig. 9: Oscilloscope screenshots of two events and the logical signals used to build the trigger scheme; a $\gamma$ event is shown in the top plot and a neutron event at the bottom.

- due to high light sensitivity almost the $40 \%$ of events are recorded in coincidence.

An advanced triggering scheme taking into account the signal amplitude and its time development was studied and implemented (Fig. 9). It essentially consists in requiring the coincidence between:

- a delayed gate generated when the leading edge of the signal exceeds a first threshold $T h_{l}$;

- a logical signal resulting from the trailing edge falling below a second threshold $T h_{t}$.

Efficiency and $\gamma$ rejection power were optimized as a function of the four parameters: $T h_{l}, T h_{t}$, gate delay and gate length. The results are reported in Table III, showing a remarkable improvement with respect to the simple leading-edge based scheme and reducing by a factor $\approx 1000$ the counting rate between ${ }^{252} \mathrm{Cf}$ and ${ }^{60} \mathrm{Co}$ sources of similar activity. This result can be turned into a $\gamma$ sensitivity at the $10^{-6}$ level once the detector acceptance is accounted for.

Preliminary sensitivity tests were performed as well with 
a ${ }^{252} \mathrm{Cf}$ source of $370 \mathrm{KBq}$ activity, recording a $62 \mathrm{~Hz}$ rate in contact, dropping to $2.7 \mathrm{~Hz}$ at $20 \mathrm{~cm}$ and $0.5 \mathrm{~Hz}$ at $50 \mathrm{~cm}$ distance. These values, once the $\gamma$ rejection power is accounted for, confirm the potential of the system and represent a significant starting point towards a full qualification with respect to the standards.

\begin{tabular}{|c|c|c|c|}
\hline & \multicolumn{3}{|c|}{ Counting frequency [Hz] } \\
\cline { 2 - 4 } & No source & ${ }^{60} \mathrm{Co}[40.4 K B q]$ & ${ }^{252} \mathrm{Cf}[37 K B q]$ \\
\hline Single arm & 0.02 & 0.05 & 12.27 \\
Two arm coincidence & 0.01 & 0.01 & 8.61 \\
\hline
\end{tabular}

TABLE III: Counting rates in different conditions implementing the delayed coincidence trigger. Sources were in contact with the detector wall.

\section{B. Off-line Algorithms for Neutron- $\gamma$ Discrimination}

While the on-line studies assessed the system performance in terms of counting statistics, the off-line analysis had the goal of measuring the rejection power of interacting $\gamma$ and the detection efficiency of interacting neutrons.

The analyzed data sets were recorded on the base of a minimum bias trigger, requiring either of the two SiPM arrays to have a signal exceeding a threshold value set at $\approx 3$ standard deviations of the baseline distribution. As above, data sets were recorded with no radioactive source (left-over noise and ambient background), ${ }^{60} \mathrm{Co}$ and ${ }^{252} \mathrm{Cf}$. The ${ }^{60} \mathrm{Co}$ sample contains both events due to noise and to interacting $\gamma$ while the ${ }^{252} \mathrm{Cf}$ run contains events from three classes: noise, $\gamma$ and neutrons. The objective of the proposed procedure is to quantify each of the event classes for every condition, implement selection algorithms and evaluate the performance.

The proposed discriminant algorithm is based on a multivariate Bayesian approach inherited from the beauty and charm quark tagging studies in the LEP experiments at CERN [15], [16]. Two samples are presumed to be available: one of them contains events of one class only (reference sample) while the other is an unknown mixture of two classes of events (mixed sample). The method aims at measuring the composition of the mixed sample and enhance one class of events against the other. The main steps of the procedure may be summarized as follows:

- a variable with known probability distribution functions with a limited overlap between the two classes of events is identified and used to measure the original composition of the mixed sample;

- a set of $\mathrm{N}$ discriminant variables known to be uncorrelated in the reference sample is considered; their spectrum in the reference sample provides the experimental probability density function $h_{i}\left(x_{i}\right)$;

- for each variable, the cumulative distribution function is constructed:

$$
I_{i}\left(x_{i}\right)=\int_{-\infty}^{x_{i}} h_{i}\left(x_{i}\right) d x_{i}
$$

known to have a uniform distribution in $[0 ; 1]$;
- as long as the variables are uncorrelated, the quantity

$$
P=\Pi \cdot \sum_{i=0}^{N} \frac{(-\log \Pi)^{i}}{i !},
$$

where $\Pi=I_{1} \cdot I_{2} \ldots \ldots \cdot I_{N}$, may be shown to be characterized as well by a uniform distribution in $[0 ; 1]$;

- $\quad \mathrm{P}$ is calculated for every event in the mixed sample. If it results from the class of events in the reference sample, $\mathrm{P}$ is uniformly distributed. On the other hand, if it is due to the complementary class of events featuring probability density functions $h_{i}\left(x_{i}\right)$ significantly different from $h_{i}\left(x_{i}\right)$, P may be expected to be characterized by a distribution peaking on 0 or 1 depending on whether $\tilde{h}_{i}\left(x_{i}\right)$ exceeds $h_{i}\left(x_{i}\right)$ to its left-hand or right-hand side. An exemplary illustration related to one of the observables used in the current analysis is shown in Fig. 10.

Setting a threshold on the P-value is equivalent to define the Confidence Level with which the hypothesis of the current event to belong the reference class is rejected. Knowing the initial and final mixed sample composition, the selection efficiency can be evaluated.
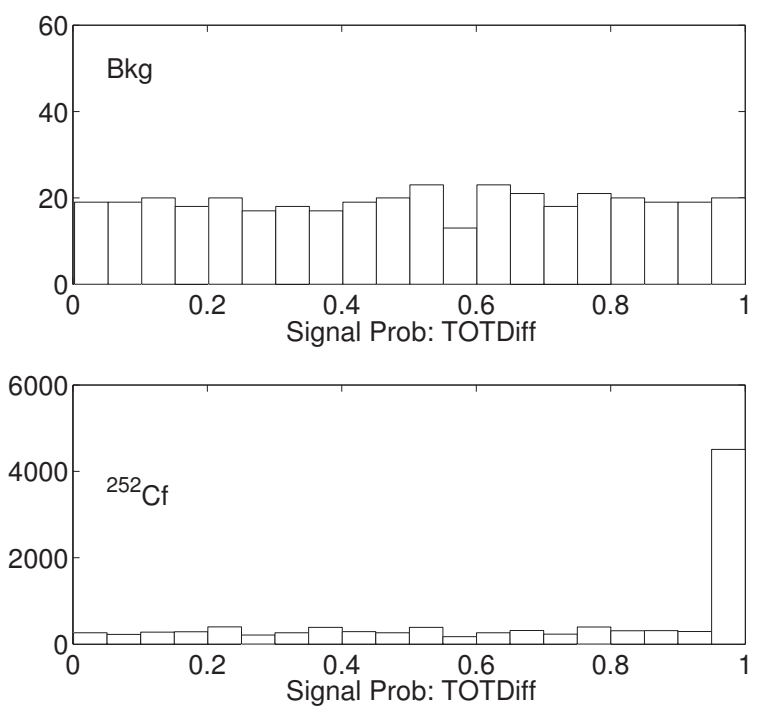

Fig. 10: Cumulative distribution function for the TOT-Diff variable used in this analysis (see text for the definition). The top plot shows the distribution for the reference sample (no source), the bottom refers to the mixed sample $\left({ }^{252} \mathrm{Cf}\right)$.

The procedure was applied twice: initially, the reference sample was the data set without a source and the goal was filtering out noise induced events in the ${ }^{252} \mathrm{Cf}$ and ${ }^{60} \mathrm{Co}$ data sets. At a second step, $\gamma$ induced events in the ${ }^{60} \mathrm{Co}$ run were the reference, with the objective of enriching the fraction of neutron events in the ${ }^{252} \mathrm{Cf}$ sample.

During the first iteration, the sample composition was determined on the base of the distribution of the signal slow 
component normalized on the total area (Fig. 11).

Once the focus was moved to neutron enhancement in the ${ }^{252} \mathrm{Cf}$ run, the Time Over Threshold (TOT) was retained as a reference distribution (Fig. 12), with TOT defined as the duration of the longest series of data above the baseline in the integration window. Concerning the discriminant variables, two quantities related to the time development of the signal were chosen, complemented by two observables linked to the deposited energy:

- the difference between the duration of the longest series of data above and below the baseline (TOT-Diff); this is expected to peak at zero for symmetric noise induced events;

- the difference between the mean value and the peak position in a pulse, i.e. a quantity related to the skewness of the distribution;

- the area of the signal, proportional to the deposited energy (Total-Charge);

- the difference between the area to the right-hand side of the signal peak and its left-hand side (Charge-Diff).

After the first iteration, about $78 \%$ and $100 \%$ of the particle induced events in the ${ }^{60} \mathrm{Co}$ and ${ }^{252} \mathrm{Cf}$ data sets were selected with a threshold value $\mathrm{P}=0.995$. Signal efficiency and background rejection power were estimated as:

$$
\begin{gathered}
\epsilon_{\text {signal }}=\frac{N_{\text {signal }}^{\text {sel }}-\epsilon_{b k g} \cdot N_{b k g}}{N_{\text {signal }}} \\
\epsilon_{\text {rej_bkg }}=1-\epsilon_{b k g}
\end{gathered}
$$

where $N_{\text {signal }}^{\text {sel }}$ is the number of events passing the selection criteria in either of the mixed samples and $N_{\text {signal }}$ is the is the original number of events of interest in the data-set. $N_{b k g}$ is the number of background events in the reference sample

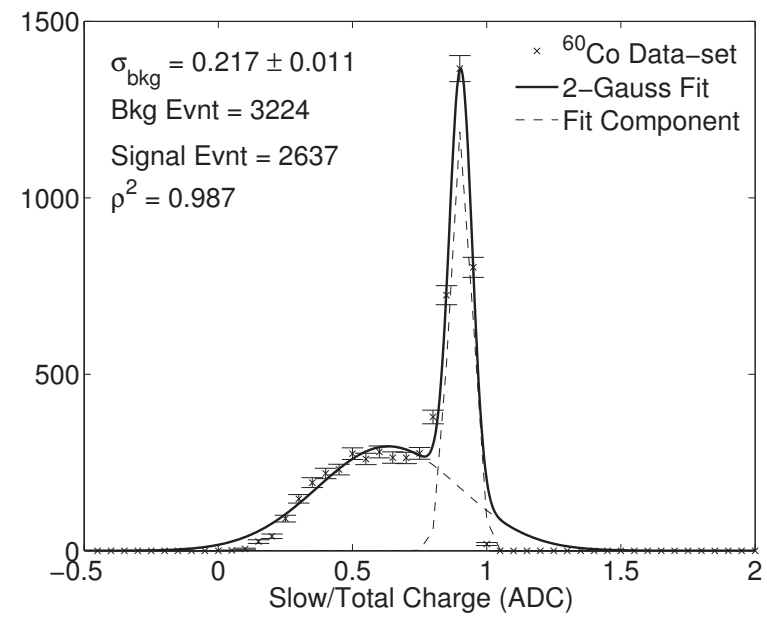

Fig. 11: Histogram of the charge integrated in the right part of the signal (slow component) over the total integrated charge for all the events in the ${ }^{60} \mathrm{Co}$ data set. The two-gaussian fit is used to estimate the number of background and signal induced events in the sample. The $\sigma_{b k g}$ obtained from the wider part of the distribution is compatible with the $\sigma$ calculated with the background data set which is $\sigma=0.202 \pm 0.007$.

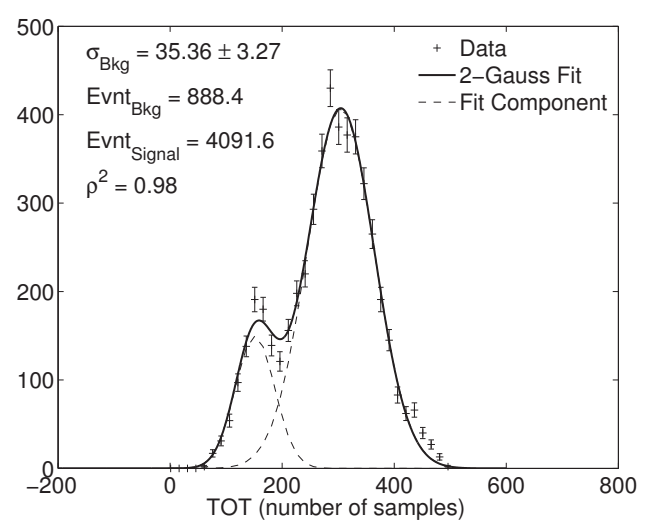

Fig. 12: Histogram of the TOT signals for all the events in the ${ }^{252} \mathrm{Cf}$ data set after having filtered the noise induced events. The two-gaussian fit is used to estimate the number of $\gamma$ and $n$ events in the sample. The $\sigma_{b k g}$ obtained from the left part of the distribution is compatible with the $\sigma$ calculated with the ${ }^{60}$ Co data set which is $\sigma=40.00 \pm 1.37$.

and and $\epsilon_{b k g}$ measures the fraction surviving the selection.

After having filtered the noise induced events, the procedure was iterated assuming the ${ }^{60} \mathrm{Co}$ data as a reference sample and aiming to reject $\gamma$ induced events in the ${ }^{252} \mathrm{Cf}$ data set. The distribution of the discriminant variables is shown in Fig. 13, after a normalization to the maximum bin content. Results are summarized in Fig. 14, showing the $n$ selection efficiency and the $\gamma$ rejection power as a function of different threshold in the $\mathrm{P}$ variable. Assuming $\mathrm{P}=0.995$, the $n$ selection efficiency is $94 \%$ with a $\gamma$ rejection power of $92 \%$, increasing to $99 \%$ with a $6 \% n$ selection efficiency reduction if the two detectors are used in coincidence.
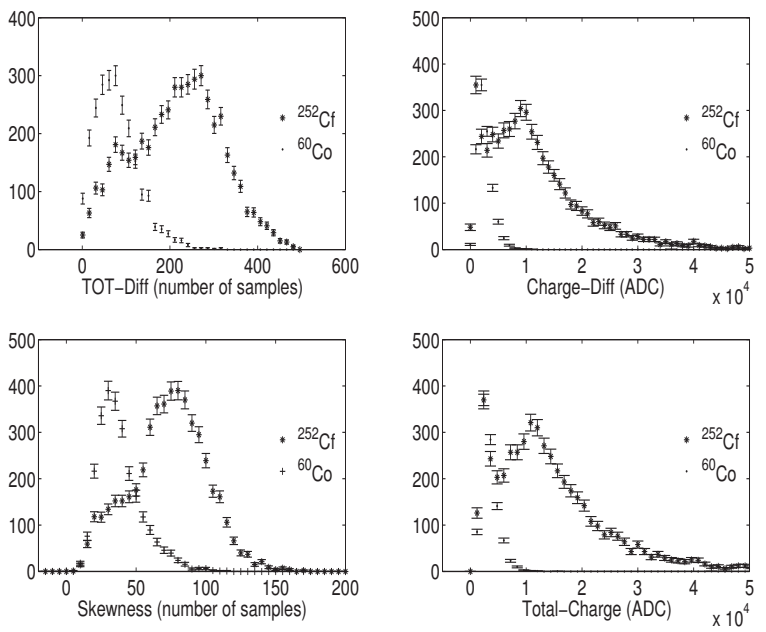

Fig. 13: Distributions of the four discriminant variables used in the second step of the procedure after having filtered the noise induced events. In all tabs the distributions obtained with the ${ }^{60} \mathrm{Co}$ and ${ }^{252} \mathrm{Cf}$ data sets are over imposed after a normalization to the maximum bin content. In all the distributions the neutron contribution is well separated with respect to the $\gamma$. 


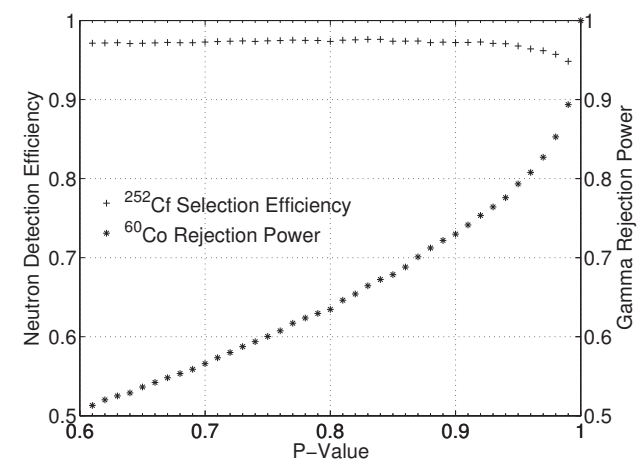

Fig. 14: Neutron selection efficiency and $\gamma$ rejection power versus the P-values.

\section{CONCLUSiOnS AND OUtLOOK}

The first results from a high pressure ${ }^{4} \mathrm{He}$ neutron detector integrating SiPM arrays were reported. In terms of selection efficiency, $88 \%$ of the detected neutrons are retained while rejecting $99 \%$ of the interacting $\gamma$. A series of tests to assess the value of these results in terms of the standards for security instruments is planned on a short timescale.

\section{ACKNOWLEDGMENT}

The reported activity was supported by the European Commission within the Seventh Framework Program, under the contract no. 284842 .

\section{REFERENCES}

[1] IAEA Nuclear Security Series No. 6, Combating Illicit Trafficking in Nuclear and other Radioactive Material, ISBN 978-92-0-109807-8, VIenna, 2007

[2] Title I of the Atomic Energy Act of 1954; see as well http://www.nrc.gov/materials/sp-nucmaterials.html and references therein

[3] V Bom, Fast Neutron Imaging for SNM Detection, SM/EN-20, Delft University, available at www-pub.iaea.org/MTCD/...CD/.../sm_en-20.pdf?

[4] S. Fetter et al., Detecting Nuclear Warheads, Science \& Global security Vol.1, no.3-4, 1990, 225-253

[5] IAEA information circulars INFCIRC/66 and INFCIRC/153

[6] R. T. Kouzes, The ${ }^{3}$ He supply problem, PNNL-18388, April 2009, prepared for the U.S. DoE, available at http://www.pnl.gov/main/ publications/external/technical_reports/PNNL-18388.pdf

[7] P. Pererani et al., Testing novel neutron detectors as alternative to ${ }^{3} \mathrm{He}$ or security applications, Nucl.Instr. Meth. A 696 (2012) 110120

[8] G. Davatz et al., Active Inspection of Nuclear Materials Using 4He Scintillation Detectors, AIP Conf. Proc. 1412 (2011) , pp. 343-350; available at http://proceedings.aip.org/resource/2/apcpcs/1412/1/343_1

[9] R. Chandra et al., Fast neutron detection with pressurized ${ }^{4} \mathrm{He}$ scintillation detectors, 2012 JINST 7 C03035 available at http://iopscience.iop. org/1748-0221/7/03/C03035

[10] R. Chandra et al., Gamma-Insensitive Fast Neutron Detector with Spectral Source Identification Potential, Homeland Security Affairs, Best Papers from the IEEE Conference on Technologies for Homeland Security (January 2011), available at http://www.hsaj.org/?article=supplement.3.2

[11] F. Resnati et al., Suitability of high-pressure xenon as scintillator for gamma ray spectroscopy, arXiv:1212.4050 [physics.ins-det]
[12] M. B. Chadwick et al., ENDF/B-VII.1 Nuclear Data for Science and Technology: Cross Sections, Covariances, Fission Product Yields and Decay Data, available at http://www.nndc.bnl.gov/exfor/endf00.jsp

[13] D. .N. McKinsey et al., Time dependence of liquid-helium fluorescence, Phys. Rev. A 67, 062716 (2003)

[14] R. T. Kouzes et al., Cosmic-ray-induced Ship-effect Neutron Measurements and Implications for Cargo Scanning at Borders, Nucl. Instr. and Meth. A 587, (2008), 89-100

[15] G. Borisov,Combined b-tagging, Nucl. Instr. and Meth. A 417 (1998) 384-390

[16] D. Buskulic et al., A precise measurement of $\Gamma_{b b} / \Gamma_{Z} \Rightarrow$ hadrons, Phys. Lett. B 313 (1993) 535-548 